\title{
THE APPLICATION SOFTWARE OF THE CERN PS ACCELERATOR CONTROLS SYSTEM - ANALYSIS OF ITS COST AND RESOURCES
}

\author{
Gianpaolo BENINCASA, Axel DANEELS, Paul HEYMANS and Christian SERRE \\ CERN, 1211 Geneva 23, Switzerland
}

\begin{abstract}
The CERN PS accelerators have evolved into one of the world's most sophisticated high energy physics facility. The variety of beams and their high repetition rate means that a most sophisticated controls system is required. This reflects on the application software. At the time of the completion of the new control system, nearly 1000 programs, amounting to around 450000 lines of code, have been developed at the cost of approximately 120 man-years. The span of this software ranges from real-time application programs to special purpose development and management tools.

This paper documents the cost, resources and production of this software project. These are analyzed in terms of the structure of the application software. Rules-of-thumb are suggested for estimating the required effort at various phases of the project and to define the implementation strategy.
\end{abstract}

\section{Introduction}

To the knowledge of the authors this application software project for accelerator controls is the first one to have established right from the start a detailed account of its implementation in order to analyse how the effort was spent. This led to the definition of heuristic rules which are now applied to evaluate any new application software by the PS controls group. Though they seem to yield reasonable results, they are likely to undergo further refinement. In the course of the project, the estimating and accounting techniques went through a learning phase; hence the later accounts are more accurate and later module inventories more complete than the earlier ones. Further, the figures are not as accurate as one would wish, because it is difficult to cross check estimates with the actually expended effort: quite often several programs are being developed in parallel, and people are reluctant to present a detailed account of their activities. Similarly, the defintion of priorities and milestones went through several iterations before settling. Nevertheless, within the frame of the CPS controls, the sample is large enough for lessons to be learned and rules derived and refined which may apply to similar projects in similar environments.

The figures and rules of thumb that are given in this paper are very much dependent on the characteristics of the CPS accelerators, the operational philosophy, and both the economic and the work conditions. All these aspects have conditioned the structure of the application software, and thus also the effort for its implementation.

\section{The CPS accelerator complex and its operation}

The CERN PS accelerator complex comprises 2 linear accelerators (Linac I and II), a 4 ring synchrotron booster (PSB), a proton synchrotron (PS), an antiproton accumulator (AA), various beam transfer lines (TT) and a low energy antiproton ring (LEAR) (fig. 1). It provides various high-energy physics experiments and other accelerators on the site with beams of different particles in pulses of $1.2 \mathrm{~s}$. The beam characteristics are modified from one pulse to another ("pulse to pulse modulation") in a repeated sequence called "supercycle" which is changed daily, if not hourly, by the operators [1]. Any beam of the supercycle in any accelerator or part of accelerator, is operated concurrently and in a similar fashion from any of 7 identical general purpose consoles in the main control room [2]. Similar consoles, of reduced format, are located in remote areas for local experiments.

Linac I and PS are the oldest machine. Their performance was gradually improved by grafting subsequent generations of more modern technologies. The other extreme is the AA, whose controls hardware benefits from up to date developments. The CPS complex is thus characterized by a mix of technologies.

The new control system, which covers the PSB, PS, AA and TT, but not the linacs or LEAR at present, is based on a network of around 25 minicomputers and more than 100 microcomputers interfaced to the process hardware through serial CAMAC (fig. 2). The minicomputers are Norsk Data machines with a multi-program operating system SINTRAN III. There are three categories: the console driving computers, one per console; front-end computers, one per accelerator; and 


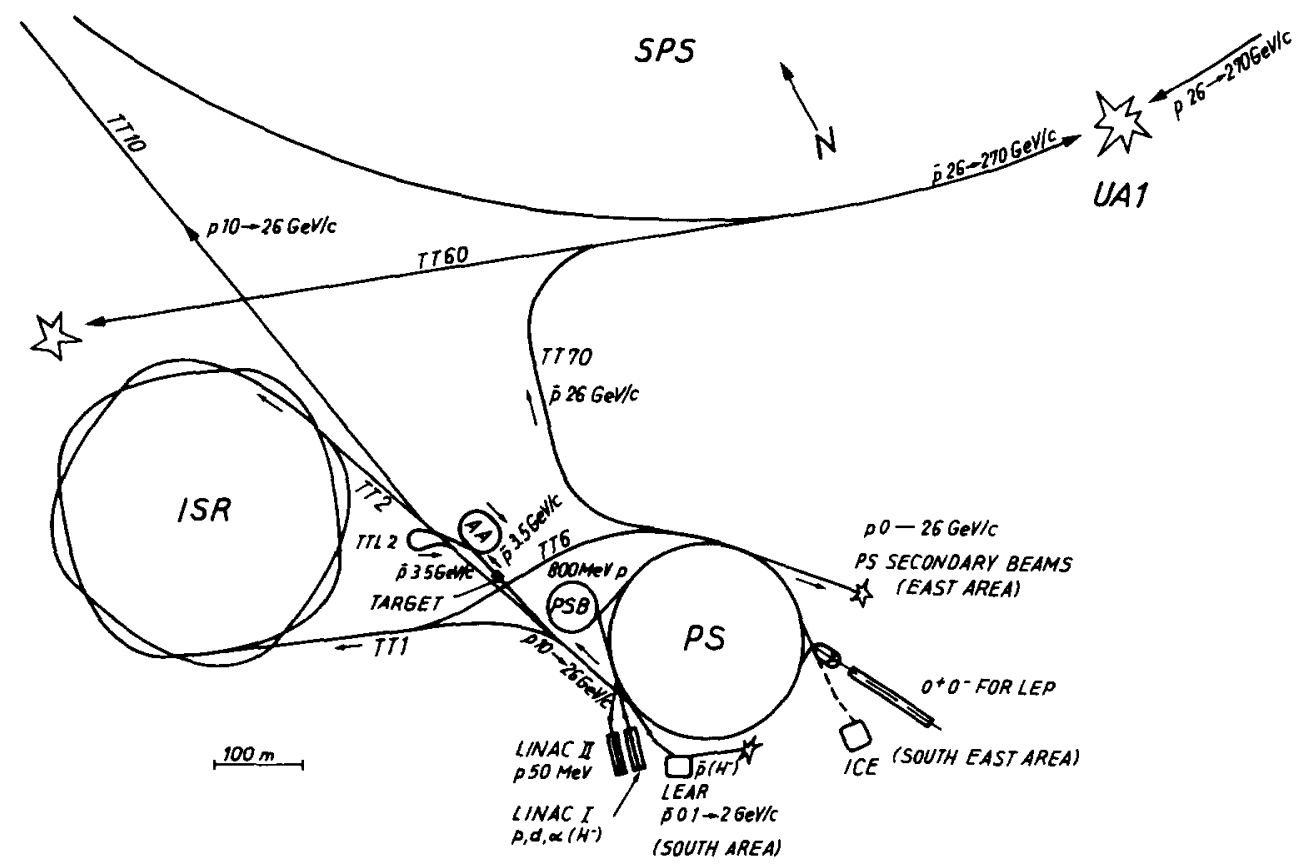

Fig. 1. Overall layout of the CPS complex.

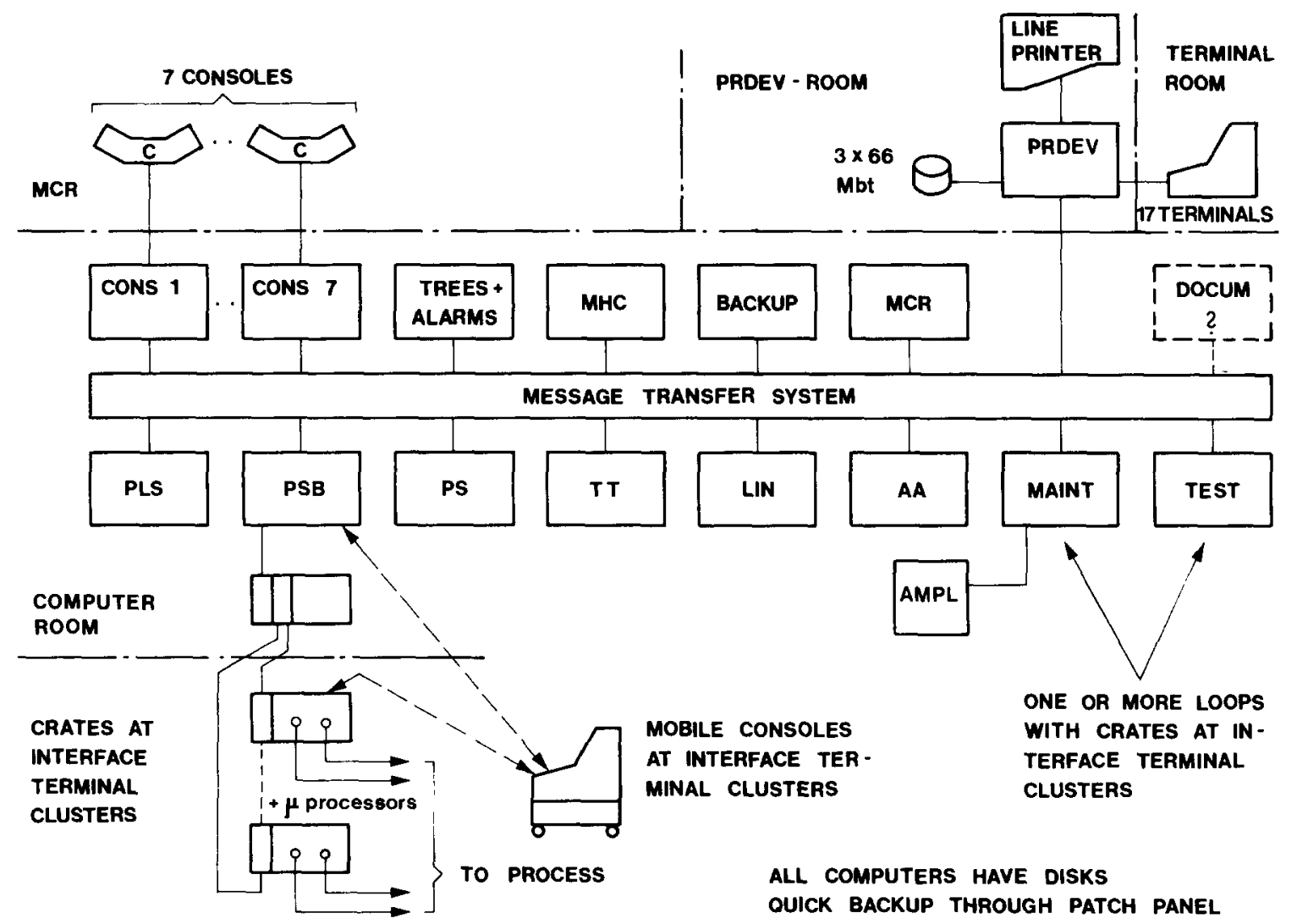

Fig. 2. Computer network. 
central activity computers for beam sequencing and synchronization, the message handler, etc. The microcomputers are TMS 9900 located in CAMAC crates as auxiliary crate controllers. They execute real time activities, such as pulse-to-pulse modulation and local data buffering. The programming languages are: ASM for the micros; the manufacturer provided intermediate level language N-PL and the home made $\mathrm{P}+$, an extension of PASCAL, for the minis; and the interpreter NODAL for tests, experiments and nontime-critical applications [3].

\section{Application software structure}

In the CERN PS terminology, system software embraces all operating systems, network software, programming languages, etc. Application software encompasses all aspects of process controls ranging from operator interaction and displays to process and equipment control algorithms and data processing.

The structure of the application software has been described elsewhere [4] and is only briefly repeated here for the sake of readability (fig. 3 ).

\section{1. "Horizontal" description}

The application software is structured in hierarchical layers of modules. Starting from the process hardware, the layers are defined as follows:

RT: Real-time Tasks, servicing hard real time events,
IM: Interface Modules, hiding the protocols of the CAMAC hardware modules,

EM: Equipment Modules, hiding the details for controlling different types of equipment.

CVM: Composite Variable Modules, which handle beam physics parameters,

PM: Process control Modules,

OM: Operator's interaction Modules, holding a dialogue with the operator.

The RT run in the micros; IM, EM, CVM and PM in the front-end minis; $\mathrm{OM}$ in the Console Computers. The RT, IM and EM levels reflect the equipment hardware; the CVM and PM ones, the beam physics; and the $\mathrm{OM}$ level represent the operation.

Finally, general purpose programs, GPPs, perform either ancillary activities like reserving sets of equipment to prevent conflicting controls between several operators, presenting $\mathrm{PM}+\mathrm{OM}$ menus available for selected accelerator subsets, knob control etc.

\section{2. "Vertical" description}

Except for the low level RT, all modules are passive and are executed under supervision of managerial tasks. The collection of these tasks is called the Skeleton: it hides the peculiarities of the accelerator processes (synchronization with cycles within the supercycle, synchronization with proper beam events and so on) of their operation (general purpose character of the consoles, etc.) and of the control system itself (network, hierarchical structure, etc.). These aspects are thus transparent to the operator and also to the application programmer.

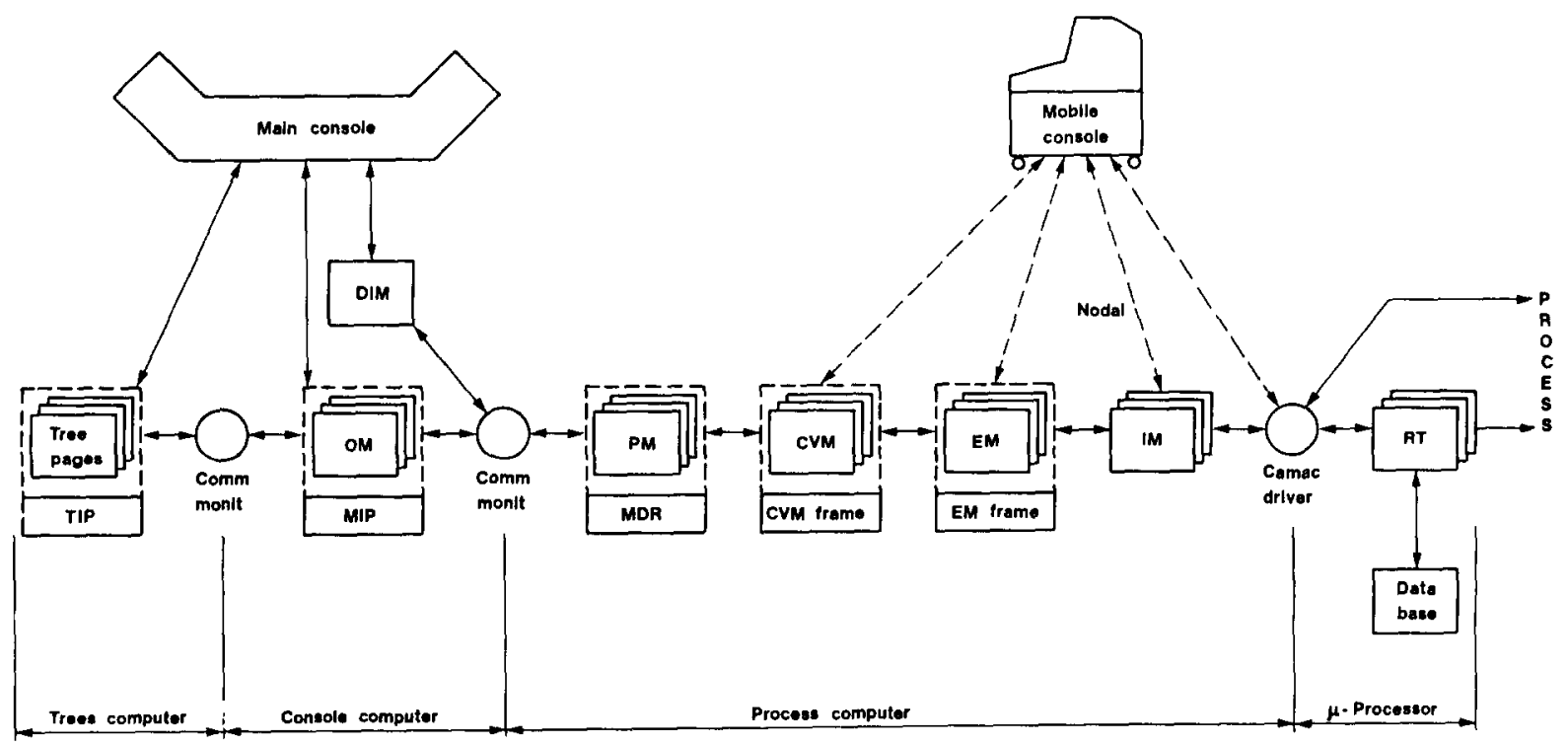

Fig. 3. Simplified software layout. 
The Skeleton and associated application modules constitute the basic structure of the application software. All control functions are constructed along that model. In particular there are a number of Basic Facilities both for hardware control and for operation. They are called "basic" because they apply to any accelerator of the CPS complex. Their degree of generality depends on the degree of standardization which is achieved for interfaces, control protocols of the various devices and operational procedures. In terms of application software, these facilities are provided by modules on various levels of the hierarchy and are common to all accelerators. The Basic Control Facilities ensure pulseto-pulse modulation of the beam characteristics, control of all CAMAC modules, power supplies and timing units, analog function generators, etc. The Basic Operational Facilties include trees to call programs, analog and video signal observation, interactive tools (knobs, touch panel, tracker ball, etc.), beam sequencing and alarm reporting. The Basic Operational facilities also includes a central data base that contains information for all data driven programs: surveillance and alarm reporting, global operations such as setting-up, archives, etc.

Skeleton and Basic Facilities build up the controls Kernel which provides a substantial portion of the facilities required for the operation of any accelerator of the CPS complex.

The bulk of the application software involves those applications which are Specific to every accelerator. They are mainly developed through appropriate production Tools (software templates, editors, listers, etc.) as standard subroutines or data files and embedded within the Skeleton.

The project size and constraints also required managment Tools for planning and progress monitoring to be developed.

\section{Distribution of modules and the effort required}

Table 1 gives the number of modules and the corresponding effort in man months (m-m) for each level of the application software structure required by each accelerator for its operation. Table 1 should thus be considered as a breakdown of the number of modules and their corresponding effort in the current application structure, as if there were an independent controls project for each accelerator. All modules which build up the Kernel are common to each machine as a result of standardization, architecture and software design. The column PLS refers to the Program Line Sequencer which the supercycle information for all machines.

This table depicts the situation that prevailed at the end of the CPS controls project, i.e. when all the PS RF was put on line. At that time the PSB had been in operation with the new system for four years, and the PS for two years, except for its RF. The figures are thus representative of the cost of a control system that provides "comfortable" operation of these accelerators. The effort is evaluated in man-months: one man-month $(\mathrm{m}-\mathrm{m})$ is taken as 20 man-days, and 1 man-year as 10 man-months, so as to take normal working hours and holidays into account. The REAL EFFORT, (E-real), covers all phases of the implementation cycle of every module starting from the early specification to the final delivery of an operational product. It includes education of newcomers who in general became productive after 3 months of training. It also includes documentation, correction of errors, modification and addition if requested by operation, and optimization of performance. However it does not include daily exploitation and maintenance.

The average size of the modules is around 400 lines of code, not including comments. At a first glance, it appears that the PSB and the PS require approximately

Table 1

Distribution of modules and effort for each accelerator

\begin{tabular}{|c|c|c|c|c|c|c|c|c|c|c|c|c|c|c|c|c|}
\hline \multirow[t]{2}{*}{ Layer } & \multicolumn{5}{|c|}{$\begin{array}{l}\text { Number of modules } \\
\text { per accelerator }\end{array}$} & \multicolumn{5}{|c|}{$\begin{array}{l}\text { Real effort (E-real) } \\
\text { per accelerator } \\
\text { (in man-months) }\end{array}$} & \multicolumn{5}{|c|}{$\begin{array}{l}\text { Effort per module (E-mod) } \\
\text { per accelerator } \\
\text { (in man-months) }\end{array}$} & \multirow[t]{2}{*}{$\begin{array}{l}\text { Average } \\
\text { effort } \\
\text { (in } \mathrm{m}-\mathrm{m} \text { ) }\end{array}$} \\
\hline & $\overline{\text { PSB }}$ & PS & $\mathrm{AA}$ & TT & PLS & $\overline{\mathrm{PSB}}$ & PS & AA & TT & PLS & PSB & PS & $\mathrm{AA}$ & TT & $\overline{\text { PLS }}$ & \\
\hline RT & 32 & 48 & - & 27 & 9 & 46 & 63 & - & 25 & 21 & 1.45 & 1.31 & - & 1 & 2.33 & 1.52 \\
\hline IM & 11 & 11 & 11 & 11 & 2 & 11 & 11 & 11 & 11 & 1 & 1 & 1 & 1 & 1 & 0.5 & 0.9 \\
\hline EM & 36 & 45 & 43 & 25 & 9 & 72 & 80 & 41 & 41 & 13 & 2 & 1.8 & 1 & 1.64 & 1.44 & 1.58 \\
\hline CVM & 2 & 20 & - & 1 & - & 2 & 14 & - & 2 & - & 1 & 0.7 & - & 2 & - & 1.23 \\
\hline $\mathbf{P M}+\mathbf{O M}$ & 289 & 305 & 181 & 185 & 56 & 405 & 449 & 245 & 285 & 98 & 1.4 & 1.5 & 1.35 & 1.5 & 1.75 & 1.5 \\
\hline Total 1 & 370 & 429 & 235 & 249 & 76 & 536 & 616 & 297 & 364 & 133 & 1.45 & 1.44 & 1.26 & 1.46 & 1.75 & 1.47 \\
\hline Tools & 24 & 22 & 21 & 22 & - & 52 & 45 & 42 & - & & & & & & & \\
\hline Total 2 & 394 & 451 & 256 & 271 & 76 & 588 & 661 & 339 & 409 & 133 & & & & & & \\
\hline
\end{tabular}


Table 2

Ratio of the number of modules to drive the equipment to the total number of modules for each machine

\begin{tabular}{lccccc}
\hline & PSB & PS & AA & TT & PLS \\
\hline Total 1 & 370 & 429 & 235 & 249 & 76 \\
RT + EM & 68 & 93 & 43 & 52 & 18 \\
Total 1 & 5.4 & 4.6 & 5.5 & 4.8 & 4.2 \\
\hline RT + EM & & & & & \\
\hline
\end{tabular}

the same number of modules, and thus also a similar effort. Next, the figures for the PSB, PS, TT and PLS suggest that these figures relate to the variety, rather than the absolute quantity, of equipment which are represented by corresponding EMs. Further, the number of RTs is equivalent to the number of EMs: each equipment that has real-time constraints has a corresponding real-time task. The RTs appear as a measure of the real-time constraint which is a dominant cost driving factor for the controls project.

The AA is different: it is operationally a simpler machine, an accumulator, with no specific real-time requirements. The AA is also new and still fairly experimental and does not have as long an operational tradition as the other machines. Moreover, despite PS's divisional policy to standardize all CPS controls, it never succeeded in imposing this policy on the AA, except for its basic configuration and equipment level software. Part of the AA beam physics programs are still being developed by ad hoc programmers; their programs are not accounted for in the official inventory which is therefore incomplete.

If one excludes the Tools, which are not really part of the controls software, and the IM as not representative of the overall accelerator process (the various equipment can in general be interfaced through a limited number of hardware modules), then, as can be seen from table 2, the number of modules to drive the equipment, i.e. the RT and EMs, represent $20 \%$ of the total number of modules to be developed (Total 1 of table 1).

From table 2, a reasonable estimate for the total number of modules requested for the controls of an accelerator is given by:

total $=5(\mathrm{EM}+\mathrm{RT})+\mathrm{IM}$.

With the assumption that, for time critical projects like the PSB, PS, TT and PLS, every equipment requires a corresponding RT, (1) yields:

total $=10 \mathrm{EM}+\mathrm{IM}$,

which gives an estimate of the size of the application package, as soon as the full list of all types of devices of an accelerator is known.

Though (1) seems also to apply to a non-time-critical machine such as the AA, in which case RT is 0 , the data for its controls project is insufficient to be more definite.

The average development effort per module for an overall application project is around 1.47 man-month. All modules are in the range of 1.31 to 2.33 man-months except for the CVMs and IMs. The IMs are rather rudimentary and merely include the most commonly used functions of every CAMAC module, hence their low cost. Using the average figure, the total effort in man-months, can then be evaluated approximately as soon as the total number of modules is estimated.

Table 3 shows that estimates based on above assumptions come reasonably close to the actual figures, the error generally being on the right side (i.e. estimate greater than actual).

\section{Breakdown of the modules and effort taking into account the structure}

Because of the large size of the project, its iterative implementation strategy and its economic and personnel constraints, a significant effort was invested to reduce its overall size and to make the production of large quantities of software modules more cost effective. Standard control protocols were defined for several categories of equipment (power supplies, timing, vacuum, etc., resulting in a library of standard software modules to control these types of equipment. This library is common to all accelerators and is part of the standard controls facilities provided by the Kernel. Similarly

Table 3

Comparaison between estimated and real number of modules and effort

\begin{tabular}{|c|c|c|c|c|c|c|c|c|}
\hline \multicolumn{4}{|c|}{ Number of modules per accelerator } & \multicolumn{5}{|c|}{ Effort per accelerator (in man-months) } \\
\hline PS & AA & TT & PLS & PSB & PS & $\mathbf{A A}$ & TT & PLS \\
\hline 461 & 226 & 261 & 92 & 545 & 677 & 332 & 383 & 135 \\
\hline 429 & 235 & 249 & 76 & 533 & 616 & 297 & 362 & 133 \\
\hline+7.5 & -4 & +5 & +21 & 2 & 10 & 12 & 6 & 1 \\
\hline
\end{tabular}

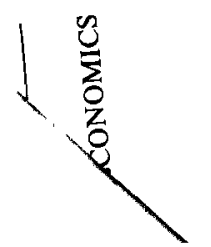


the standardization of operational procedures resulted in standard data driven GPPs.

Special purpose Tools were developed to increase the productivity, reliability and maintainability of the software modules, and to aid project management.

One of the difficulties of a large project is that it requires many programmers and this entails more human communication overhead. This was overcome by a staff organization that reflects the project structure [5].

Table 4 shows the distribution of the software modules taking into account the application structure. It shows how many modules were developed on each level of the hierarchy, specific to each accelerator, and how many build up the Kernel, common to all machines.

A comparison between tables 1 and 4 illustrates the economy that has been realized. This is primarily due to the Kernel which represents $23 \%$ of the total application package. The low level Kernel software, i.e. the equipment level RTs, IMs and EMs, result from the hardware standardization. The high level Kernel modules, CVMs, PM + OM's and GPPs result from the definition of a single operational philosophy for all machines.

Whereas the PSB uses 95\% of the Kernel library, the PS $99 \%$, the AA $62 \%$, the TT $87 \%$, the PLS only benefits from $4 \%$ of this library. This indicates that the software design was basically accelerator oriented, and that the central PLS system was more of a stand alone project that did not quite fit into the standard frame. The GPP modules that had to be developed specifically for each individual accelerator are essentially data files describing the specific machines and used by corresponding data driven Kernel programs. The Tools which were developed both for production and management only represent of $2.6 \%$ of the total software package. How much savings they realize can be measured from the development effort and later maintenance effort.

Table 5 compares the effort which was originally estimated (E-est.) for all modules in each layer with the effort actually spent (E-real). This estimate was based on the experience with the old controls and more recently with a 12 man-year pilot project that was set up prior to the actual CPS controls project, to evaluate the applicability of the SPS control system to the CPS specific environment, and to gain experience with software engineering techniques [6]. In general every module was discussed with its programmer and an estimated effort agreed upon. The real effort, i.e. the one actually spent, includes correction for underestimates, design errors, modification to the original design and later extension.

The real effort for the overall application project exceeds the estimate by $17 \%$ (table 6 ): i.e. roughly $4 \%$ correction, $10 \%$ modification and extension and $3 \%$ optimization of performance.

Generally speaking the estimates were best for the accelerator control subprojects. However the effort for the PSB controls was higher than estimated, because it was the first subproject in the implementation sequence. The overall project engineering and management had not reached the maturity yet as for the subsequent subprojects.

The PLS and Kernel part of the controls were severely underestimated because the implications of their central key position in the system was not fully appreciated. In particular the real-time constraints, the robustness and the user-friendliness of the PLS became fully apparent only after this system came into operation. The same consideration apply to the Kernel software which accommodates all exotic operational requirements: virtual accelerators, anonymous consoles, sharing of displays between several programs, multiplexed use of meausurement devices, etc. The routines need to be fast and were therefore mostly written in N-PL; also they should be most reliable. Evidently they turned out more complex and hence more effort consuming than anticipated. In addition the Kernel absorbed most of the effort required for optimizing the performances of the overall system.

This is also evident from table 7 , which shows that

Table 4

Distribution of modules per accelerator in the application structure

\begin{tabular}{|c|c|c|c|c|c|c|c|c|}
\hline \multirow[t]{2}{*}{ Layer } & \multicolumn{8}{|c|}{ Number of modules developed per accelerator } \\
\hline & PSB & PS & AA & TT & PLS & Global & Kernel & Total \\
\hline$\overline{\mathrm{RT}}$ & 14 & 27 & - & 9 & 9 & 59 & 21 & 80 \\
\hline IM & - & - & - & - & - & - & 11 & 11 \\
\hline EM & 23 & 32 & 38 & 17 & 6 & 116 & 15 & 131 \\
\hline CVM & - & 18 & - & 1 & - & 19 & 2 & 21 \\
\hline $\mathrm{PM}+\mathrm{OM}$ & 147 & 152 & 85 & 62 & 48 & 494 & 17 & 511 \\
\hline GPP & 8 & 12 & 3 & - & 4 & 27 & 124 & 151 \\
\hline Tools & 2 & - & - & - & - & 2 & 22 & 24 \\
\hline Total & 194 & 241 & 126 & 89 & 67 & 717 & 212 & 929 \\
\hline
\end{tabular}


Table 5

Comparison between estimated and real effort within the structure

\begin{tabular}{|c|c|c|c|c|c|c|c|c|c|c|c|c|c|c|c|c|}
\hline \multirow[t]{2}{*}{ Layer } & \multicolumn{8}{|c|}{$\begin{array}{l}\text { Estimated effort per accelerator } \\
\text { (E-est. in } \mathrm{m} \text {-m) }\end{array}$} & \multicolumn{8}{|c|}{$\begin{array}{l}\text { Real effort per accelerator } \\
\text { (E-real in } \mathrm{m}-\mathrm{m} \text { ) }\end{array}$} \\
\hline & PSB & PS & $\mathrm{AA}$ & TT & PLS & $\begin{array}{l}\text { Glo- } \\
\text { bal }\end{array}$ & $\begin{array}{l}\text { Ker- } \\
\text { nel }\end{array}$ & Tot. & PSB & PS & $\mathrm{AA}$ & TT & PLS & $\begin{array}{l}\text { Glo- } \\
\text { bal }\end{array}$ & $\begin{array}{l}\text { Ker- } \\
\text { nel }\end{array}$ & $\overline{\text { Tot. }}$ \\
\hline$\overline{\mathrm{RT}}$ & 29 & 41 & - & 9 & 10 & 89 & 21 & 110 & 31 & 41 & - & 10 & 21 & 103 & 22 & 125 \\
\hline IM & - & - & - & - & - & - & 6 & 6 & - & - & - & - & - & - & 11 & 11 \\
\hline EM & 35 & 52 & 31 & 22 & 7 & 147 & 30 & 177 & 42 & 52 & 32 & 23 & 8 & 157 & 37 & 194 \\
\hline CVM & - & 13 & - & 2 & - & 15 & 2 & 17 & - & 14 & - & 2 & - & 16 & 2 & 18 \\
\hline $\mathrm{PM}+\mathrm{OM}$ & 125 & 195 & 62 & 68 & 49 & 499 & 22 & 521 & 160 & 197 & 63 & 72 & 86 & 578 & 26 & 604 \\
\hline GPP & 7 & 7 & 1 & - & 2 & 17 & 165 & 182 & 5 & 7 & 1 & - & 2 & 15 & 219 & 234 \\
\hline Tools & 4 & - & - & - & - & 4 & 43 & 47 & 7 & - & - & - & - & 7 & 45 & 52 \\
\hline Total & 200 & 308 & 94 & 101 & 68 & 771 & 289 & 1060 & 245 & 311 & 96 & 107 & 117 & 876 & 362 & 1238 \\
\hline
\end{tabular}

Table 6

Estimation errors broken down by machine*

\begin{tabular}{|c|c|c|c|c|c|c|c|c|}
\hline & \multicolumn{5}{|c|}{ Accelerators } & \multirow[t]{2}{*}{ Global } & \multirow[t]{2}{*}{ Kernel } & \multirow[t]{2}{*}{ Total } \\
\hline & PSB & PS & $\mathrm{AA}$ & TT & $\overline{\text { PLS }}$ & & & \\
\hline$\frac{\text { E-real }}{\text { E-est. }}$ & 1.22 & 1.0 & 1.0 & 1.04 & 1.73 & 1.13 & 1.26 & 1.17 \\
\hline
\end{tabular}

the average effort requested for all PLS and Kernel modules was respectively 1.75 and 1.7 man-month, whereas the accelerator projects only required 1.3 manmonth per module on average.

Within the accelerator specific packages, RT and EM were not unexpectedly the more expensive because of their real time and reliability constraints, and the programming language (N-PL for EMs, ASM for RT). Production of RTs suffered from the restricted microcomputer configuration. In general the accelerator specific RTs were on average more expensive than the Kernel ones because the beam instrumentation was not standardized. The high cost of EM in the Kernel soft- ware reflects the CPS wide effort to standardize power supply and timing control despite their varied technologies. Though they were interfaced through identical CAMAC modules, one often had to merge into a single software module slight variations in the controls of similar equipment. As a matter of fact, more often than not the standardization of equipment was only achieved in the EMs. The saving that can be obtained from a standard and modern technology is best illustrated by the low cost of the AA modules. Because of these aspects, the PLS and Kernel modules were allocated to the most experienced programmers of the application group.

Table 7

Average effort per module within the application structure

\begin{tabular}{|c|c|c|c|c|c|c|c|c|}
\hline \multirow[t]{2}{*}{ Layer } & \multicolumn{8}{|c|}{ Average effort per accelerator (E-aver. in $m-m$ ) } \\
\hline & $\overline{\text { PSB }}$ & PS & $\overline{A A}$ & TT & PLS & Global & Kernel & Total \\
\hline$\overline{\mathrm{RT}}$ & 2.2 & 1.5 & - & 1 & 2.3 & 1.7 & 1 & 1.6 \\
\hline IM & - & - & - & - & - & - & 1 & 1 \\
\hline EM & 1.8 & 1.6 & 0.8 & 1.4 & 1.3 & 1.4 & 2.5 & 1.5 \\
\hline CVM & - & 0.8 & - & 2 & - & 0.8 & 1 & 0.9 \\
\hline $\mathrm{PM}+\mathrm{OM}$ & 1.1 & 1.3 & 0.75 & 1.2 & 1.8 & 1.2 & 1.5 & 1.2 \\
\hline GPP & 0.6 & 0.6 & 0.3 & - & 0.5 & 0.5 & 1.8 & 1.6 \\
\hline Tools & 3.5 & - & - & - & - & 3.5 & 2 & 2.2 \\
\hline Total & 1.3 & 1.3 & 0.75 & 1.2 & 1.75 & 1.2 & 1.7 & 1.3 \\
\hline
\end{tabular}


Beam physics programs have more relaxed constraints. Although the first ones were developed in $\mathrm{N}$-PL, the later ones benefitted from $\mathrm{P}+$. Others, nonrepetitive display programs and test programs were written in NODAL. They could therefore be implemented by temporary programmers, as imposed by the personnel reduction policy of the organization. The high personnel turnover explains why the effort for these modules is still fairly high compared to the equipment level modules, despite their relative simplicity, the transparency of the low level software and the numerous special purpose production Tools.

Tools need essentially to be user friendly, reliable and foolproof, but they are not constrained by real time and execution time. They are quite independent of one another and were also implemented by temporary programmers: hence their high cost in manpower.

\section{Implementation in slices}

The CPS controls project was implemented in around seven years, from early 1978 to early 1985 . Three major slices were defined based on operational considerations [5]. The first slice encompassed the design of the overall system, the implementation of the PSB controls and the AA equipment level controls. Obviously this slice also had to include the PLS, most of the Kernel software and the Tools. The second slice involved the PS controls except for its RF. The third slice involved the PS-ejection and beam transfer (TT), the AA beam level application and the PS-RF.

Together with the operation staff, each slice was then broken up into packages with priorities ranging from 1 to 3. The first priority package was defined as that which would allow rudimentary operation of the accelerators: i.e. trees to select programs and equipment from any of the consoles in the main control room, knob controls of individual equipment, analog and video signal observation, simple alpha-numeric display programs showing lists of equipment and their corresponding set and read back values, data logging. The second priority package included all instrumentation with more sophisticated display programs and alarm reporting. The third priority was defined as "comfortable" operation by providing controls involving entire subsets of accelerators, comparison of settings, automated set-up sequences, archives with reproduction of archived working conditions, statistics, optimization, etc.

The three priority packages are of approximately equal load in each slice (table 8).

The implementation of the slices was primarily dictated by the scheduled long machine shutdowns in which the new controls hardware could be installed, and conditioned by the available manpower. Whereas the first and second priority packages had to be implemented if the machine were to be operational, the third priority package gave some leaway to accommodate changes in the implementation strategy in case of deficiencies. The first and second priority packages were considered a must. The third priority package was implemented alongside the first and second one of the next slice. This yielded packages of approximately the same size, around 40 man-years, which was commensurate with the manpower, and allowed Operation to get hands on experience with the new controls before starting the production of the rather expensive comfort programs.

The high cost of the first slice third priority package is essentially due to corrections, $5 \%$, and supplementary requests and optimization of performances, $20 \%$. These figures rapidly decrease from the next slice onwards where only $0.5 \%$ correction and $6 \%$ supplement are recorded. The last slice has virtually no correction and only $2 \%$ of modification and supplementary requests. This indicates the experience which both programmers and Operation has accumulated during the implementation, and the stability which Kernel and Tools had reached.

\section{The "beam limit"}

In order to monitor the progress of the project, milestones were defined in the implementation of every module [5]:

Table 8

Breakdown of the actual effort expended by implementation slice and priority

\begin{tabular}{|c|c|c|c|c|c|c|c|c|}
\hline \multirow[t]{3}{*}{ Priority } & \multicolumn{8}{|c|}{ Real effort per slice and priority (E-real in $\mathrm{m}-\mathrm{m}$ and $\%$ ) } \\
\hline & \multicolumn{2}{|l|}{ Slice 1} & \multicolumn{2}{|l|}{ Slice 2} & \multicolumn{2}{|l|}{ Shice 3} & \multicolumn{2}{|l|}{ Global } \\
\hline & $\overline{(\mathrm{m}-\mathrm{m})}$ & $(\%)$ & $(\mathrm{m}-\mathrm{m})$ & $(\%)$ & $(\mathrm{m} \cdot \mathrm{m})$ & $(\%)$ & $(\mathrm{m}-\mathrm{m})$ & $(\%)$ \\
\hline$\overline{1}$ & 212 & 34 & 100 & 30 & 114 & 42 & 426 & 34 \\
\hline 2 & 215 & 34 & 77 & 23 & 130 & 47 & 422 & 34 \\
\hline 3 & 201 & 32 & 160 & 47 & 30 & 11 & 390 & 32 \\
\hline Total & 628 & 100 & 337 & 100 & 274 & 100 & 1238 & 100 \\
\hline
\end{tabular}


Design: i.e. system engineering, 20\%; and detailed design, 20\%;

Coding: $\quad$ i.e. coding and unit test, $20 \%$;

Testing: i.e. simulation test on a special purpose computer, 10\%; on-line test outside beam production, $10 \%$ on-line test with beam production, $10 \%$;

Documentation: although supposedly done all along the implementation, there was a $10 \%$ provision for the final update after commissioning.

Therefore, a module that is ripe for on-line test with beam, is $80 \%$ ready. This $80 \%$ "beam limit" was introduced to evaluate whether a controls slice would attain a reasonable operational stage: its first and second priority packages should reach this beam limit by the end of a machine shutdown. Alternately it allowed one to concentrate sufficient resources to meet this hard deadline, and a subsequent reduction in effort did not affect beam production.

\section{Conclusion}

The figures and rules that have been established in the course of this project are far from mature, and the sample is too small to draw hard conclusions. Also they are definitely biassed by the CPS specific environment and its particular application software structure. It would therefore be most interesting to compare these figures and rules with ones that result from other similar projects elsewhere. However within the frame of the CPS controls group, they are being applied to evaluate both the size and the effort for any new controls application packages. So far they have yielded acceptable estimates.

The general procedure is as follows:

(1) At the initial stage of the project, all types of equipment that control an accelerator are listed. In so far as the hardware interface complies with the prevailing CPS CAMAC standards, the size and the corresponding effort are estimated, following the procedure given in section 5 .

(2) Next, the operational programs are inventoried together with the operational staff, whilst the control protocols of the various devices are analysed in detail. This gives input to the controls hardware specialist to lay down the CAMAC configuration based on the standard modules.

(3) A full list of modules on every level of the application hierarchy is thus obtained, and the overall effort can be estimated by applying the figures of table 7 .

(4) Next priority packages are defined taking into account the delivery schedule and manpower.

(5) Finally the implementation strategy is established so as to attain the beam limit by the end of a shutdown.

(6) The progress is monitored at regular intervals so as not to lose sight of the beam limit.

\section{Acknowledgment}

The authors are indebted to B. Kuiper, PS controls group leader, who continuously encouraged the use of appropriate engineering methods in the course of this controls application project.

\section{References}

[1] P.P. Heymans and B. Kuiper, Concurrent control of interacting accelerators with particles of varying format and kind, presented at the EPS Europhysics Conf. on Computer in Accelerator Design and Operation, Berlin (1983).

[2] A. Gagnaire, R. Debordes and F. Perriollat, The main operator console of the PS accelerator complex, presented at the Particle Accelerator Conf. Vancouver (May 13-16, 1985).

[3] R. Cailliau, B. Carpenter, G. Cuisinier and W. Remmer, System software for the CERN proton synchrotron control system, CERN 84-16 (20 December, 1984).

[4] G.P. Benincasa, A. Daneels, P. Heymans and Ch. Serre, Design goals and application software structure for the CERN $28 \mathrm{GeV}$ accelerator complex, presented at the 2nd IFAC/IFIP Symp. on Software for Computer Controls, Prague (1979).

[5] G.P. Benincasa, A. Daneels, P. Heymans and Ch. Serre, Engineering a large application software project: the controls of the CERN PS accelerator complex, presented at the Particle Accelerator Conference, Vancouver (May 13-16, 1985).

[6] G.P. Benincasa, A. Daneels, P. Heymans and Ch. Serre, Structured design benefits to a process control software project, presented at the ACM Sigmini Symp. on Small Systems, New York (August 2-3, 1978). 